\title{
WalkECG: A Mobile Cardiac Care Device
}

\author{
R González, D Jiménez, O Vargas \\ Central Institute of Digital Research, Cuba
}

\begin{abstract}
A small device, named WalkECG, developed for out of hospital patient monitoring is presented. This device is based on the MSP430F149 microcontroller and includes a two-channel ECG amplifier, a graphic LCD, a keypad, a GSM modem and a battery. The heart rate, the ST segment deviation and the premature ventricular beats rate are computed and a normality limit is set for each one. When one of these limits is reached, an ECG strip is transmitted to a Telemedicine Central Station (TCS) and the device waits to display the receiving answer. The methods used to analyze the ECG were tested with real ECGs and all the QRS complexes were detected. Never a ventricular beat was classified as normal and $97.67 \%$ of normal complexes were well classified. When the communication process was tested, a remarkable difference between the transmitting and receiving signals was not observed.
\end{abstract}

\section{Introduction}

Cardiac diseases are one of the most important causes of death in the world and many efforts are dedicated to solve the prevention and treatment of this kind of pathologies [1]. The health sector faces serious and increasing problems in the management of resources for cardiac disease prevention, treatment and monitoring [5]. Telemedicine is an emerging field in health care services that could have a revolutionary impact on the medical services. The use of the mobile communications, mainly de GSM/GPRS standard, is necessary when persons should be monitoring and they will be out of the hospital in their normal lives. One kind of solution to this problem is to develop portable medical devices able to monitoring and transmit vital signals such as electrocardiogram (ECG), blood pressure, etc.

Time-varying series quantity is one of the basic features of vital signals, which contain a lot of information to be handle [5]. Mobile communications are too expensive to implement a continuos signal monitoring and the detection of pathological events is a solution to reduce the quantitiy of information to transmit. Several projects have been developed to create new services that will allow the monitoring of vital signals for out of hospital patients, but a unique and standard solution is not available. The MobiHealth project is an example of this kind of efforts [5].

WalkECG is a part of a Telemedicine Cuban project, which is being developed to combine the communication and informatic tools in the health service improvement. This device have been developed to implement the continuous monitoring of some ECG variables in out of hospital patients prone to a heart attack. The GSM/GPRS standard is used to implement the signal transmision. When a dangerous cardiac event is detected, an ECG strip is transmitted to a Telemedicine Central Station (TCS) and it will reply about what should be done. The goal of this paper is to present the main characteristics of the designed device and the results up today.

\section{Methods}

The design of WalkECG was oriented to develop a device with a low cost, reliable and easy to use. Also, it must be as small as possible. The surface montage tecnology (SMT) was used in conjuntion with multilayer print circuit.and the three-volt-powered electronic components. WalkECG can be divided in the following blocks:

- The ECG amplifier: It is two-channel ECG amplifier characterized by its low power consumption (below $10 \mu \mathrm{A}$ ). The amplifier design is based on the LT1496 operational amplifier and the mcropower single-supply INA321 instrumentation amplifier. The bandwith is limited between $1 \mathrm{~Hz}$ and $30 \mathrm{~Hz}$ in order to improve the signal quality and to keep the main ECG frequency components [2].

- The MSP430F149 microcontroller: It is an ultralow-power microcontroller with a 16-bit RISC architecture designed by Texas Instruments. The microcontroller is very compact and includes, in a single chip, several peripherals such as serial ports, timers, program memory, data memory, A/D converter, etc.

- The GSM modem: A MO110 embeded modem from SAGEM was used. It includes a serial interface to communicate with a host, signals for 
the management of the SIM card and other control signals. All these signals are contained in a 60-pin board to board connector.

- The graphic LCD: The display used is the model GF5123FBWBF of Crystal Clear Technology; its graphic resolution is $128 \times 64$ dots. It is used to show several data as part of the user interface. Graphic capabilities can be used to check the signal quality when the device is connected to the patient. Also, the answers of the TCS are displayed if the patient cooperation is neccesary.

- The keypad: This peripheral is used to introduce configuration data mainly. It is composed by five soft-touch keys and it is water-protected.

- The battery: The isolated power is supplied by two $\mathrm{NiMH}$ batteries, which are recharged in a charging station when not in use.

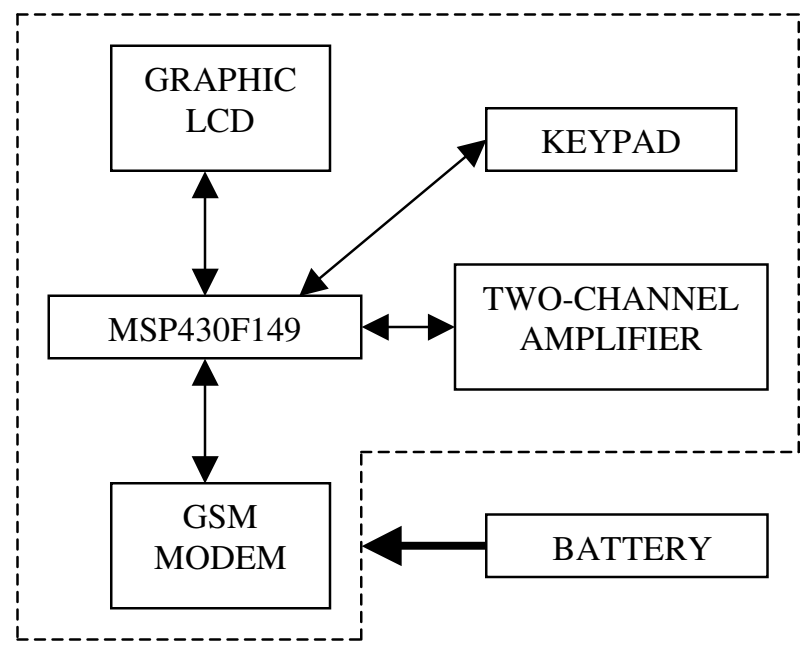

Figure 1. Block diagram of WalkECG.

The ECG amplifier, the graphic LCD, the microcontroller and other elements are mounted on a small print-circuit board, which is connected to the GSM modem with a board to board connector.

The WalkECG firmware has to analize two bipolar ECG leads in realtime in order to detect dangerous events. All the process can be divided in the following steps:

- ECG acquisition.

- QRS complex detection.

- QRS complex classification.

- ST segment deviation measurement.

- Communication.
The first step is the ECG analogue to digital conversion. Two bipolar leads are sampled simultaneously at a rate of $250 \mathrm{~Hz}$. The digital ECG is smoothed using a Hanning filter [6] and an energy function is computed sample by sample for each channel. The expression of the energy function is the following:

$$
y(k)=\frac{1}{N} \sum_{\mathrm{n}=\mathrm{k}-\mathrm{N}+1}^{k}[x(n)-x(n-1)]^{2}
$$

where

$y(i)$ : energy function

$\mathrm{x}(\mathrm{i})$ : input signal

$\mathrm{N}$ : width of the energy function

The QRS detection complex is based on the energy function and two thresholds. One threshold set the border between the high-energy peaks, associated to the QRS complexes, and the rest of the signal. The second threshold was used to set approach to the onset and offset for each QRS complex. These points are the elements used to compute the duration of each QRS complex; the candidates to be a true QRS complex should reach a minimum duration $(30 \mathrm{~ms})$. When a QRS complex is detected, its previous RR interval is computed and a RR interval average is updated. Also a baseline approach is computed as the average of the samples associated to 20 $\mathrm{ms}$ before que QRS complex onset [3, 4]. The average heart rate is updated each ten seconds.

The QRS complexes detected are classified in normal beats (NB), premature beats (PB) and unclassified beats (UB). The premature complexes rate is computed to detect when it reaches a dangerous value. Also, the ST segment deviation is calculated only in normal complexes. The classification rules should be simples because they will be applied in realtime. The rules are the following:

- The duration of a RR interval previous to a normal beat (NB) must be between $80 \%$ and $110 \%$ of the average duration of this interval.

- The duration of a normal beat must be between $85 \%$ and $110 \%$ of the average QRS duration.

- The premature beat duration must be greater than $120 \%$ of the average QRS complex width.

- The duration of a RR interval previous to a premature beat $(\mathrm{PB})$ must be less than $80 \%$ of the average duration of this interval. 


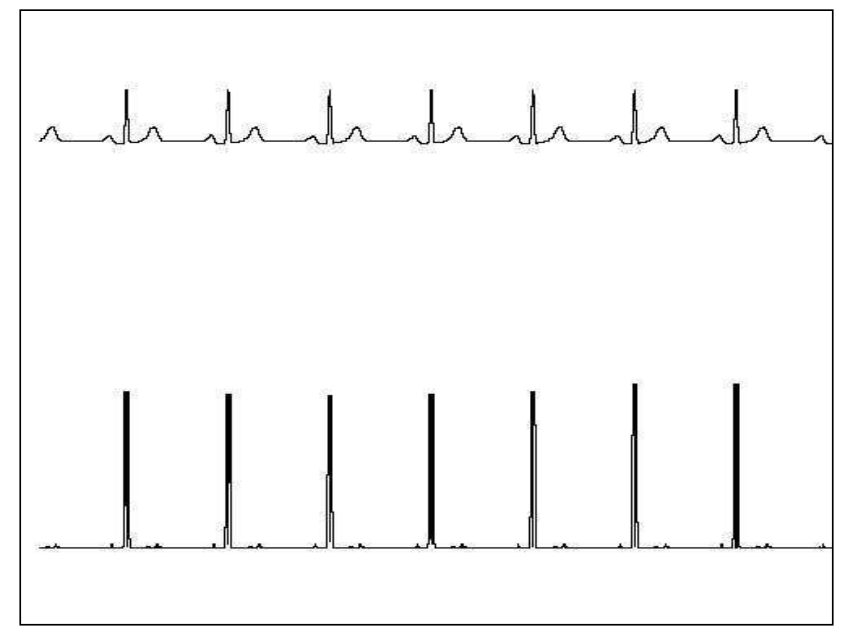

Figure 2. ECG strip and the energy function.

The premature beats rate is updated each ten second. Unclassified beats are not studied.

The ST deviation is measured in normal beats only. It is computed as the difference in voltage between the sample place $80 \mathrm{~ms}$ after the QRS complex onset and the baseline for the studied complex.

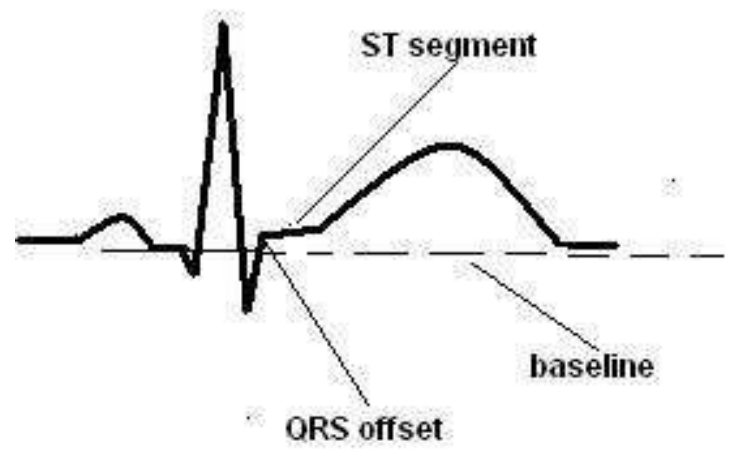

Figure 3. ST segment deviation.

When the WalkECG is turn on and configured, a first information block is transmitted to the TCS in order to open a patient profile. This block includes the following information:

- Device identification.

- Sampling rate.

- Amplitude resolution.

- Channel quantity.

- Studied variables (ST segment deviation, heart rate, etc.) and the limit for each one.

The TCS should answer with an echo block. If this block is equal to the original block, the device will be in contact with the TCS. If an error is detected in the echo block, the device will try to get the right answer three times. When a correct echo block is not received, the device displays an error message and the ECG is not processed.

Walk ECG will send ten-second ECG strips to the TCS when it detects dangerous events such as marked tachycardia or bradycardia, high premature beats rate or important ST segment deviations. The information is sent by blocks and each one has a head, a body and a tail. The head indicates the block type (identification, signal and end), the body is the data to transmit and the tail is a block checksum. The communication always starts with an identification block that informs to the TCS the cause of the transmission. The ECG strip is divided in several signal blocks and at the end a tail block is transmitted including the block quantity transmitted. WalkECG will wait for an answer, which will be displayed if the patient cooperation is necessary.

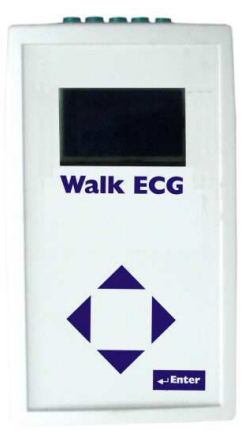

Figure 4. Front view of WalkECG.

\section{Results}

The developed method to process the ECG has been tested with twenty five-minute ECGs, including eight ECGs with premature beats. The signals were analyzed by two highly-qualified cardiologists with the help of a personal computer and several visual and graphic tools. They were not aware of the method results in order to make a blind evaluation. The opinions and conclusions of the specialists were the golden rule to test the method. A WalkECG prototype was built and the proposed method was emulated on this platform.

The evaluation results can be summarized in the following table: 
Table 1

Global results of the proposed method for realtime ECG analysis

\begin{tabular}{|l|l|}
\hline QRS complexes studied & 7268 \\
\hline QRS complexes detected & $7260(100 \%)$ \\
\hline Normal QRS complexes & 6993 \\
\hline Normal QRS well-classified & $6830(97,67 \%)$ \\
\hline Ventricular QRS complexes & 275 \\
\hline Ventricular QRS well-classified & $266(96.73 \%)$ \\
\hline
\end{tabular}

The ST measurement algorithm was not evaluated because the WalkECG first version will not included this feature. However, the algorithm was implemented in order to evaluate the real-time performance of the proposed method.

- The communication with the TCS was done when one of the following situations was present:

- More than two premature beats in ten seconds.

- Heart rate value greater than 100 beat per second (bpm).

- Heart rate value less than $60 \mathrm{bpm}$.

Forty ECG strips were transmitted and the communication process was successful for every strip. The signals received at the TCS were compared, sample by sample, with the transmitted signal. A graphic program was used for this test and remarkable differences were not observed.

\section{Discussion and conclusions}

A WalkECG prototype has been built and the proposed method for real-time ECG processing was implemented and preliminary evaluated.

All the QRS complexes were detected, it is very important because the QRS complex detection is the base to the detection of the dangerous events. It is remarkable that false positives were not observed in this detection process.

The QRS complex classification process should be based on simple rules because of the real-time implementation. The defined rules are simples and effectives, more than $96 \%$ of ventricular beats were identified. Also, the majority of normal QRS complexes were identified. A small quantity of normal complexes was misclassified, but this situation did not affect the method performance. The main goal was to detect the ventricular beats and never this kind of complex was classified as normal. Nevertheless, some of them were identified as unclassified beats (UB).
The communication process was effective, it never was aborted and the received signals were not corrupted by noise or distortions.

WalkECG looks like a useful monitoring tool for patients prone to heart attach. Also, it could be useful to long-term ECG studies. This device is the first step in the development of a family of portable devices dedicated to the Telemediicne.

\section{References}

[1] Bermúdez F. Electrocardiografía Diagnóstica, Editoral Mc Graw Hill Interamericana de Venezuela, 1998

[2] Company-Bosch E, Hartmann E. ECG Front-End Design is Simplified with MicroCoverter. Analog Dialogue 37-11, http://wwwanalog.com/analogdialogue, November 2003

[3] González R. A Spatial Study of the ST Segment. Proc. Eng. in Med. and Biol. Soc. 25th Annual Intern. Conf., Cancún México, 2003, pp. 2610-2614

[4] González R, Fernàndez R, Raola MC. Real-Time QT Interval Measurement. World Congress on Medical Physics and Biomedical Engineering. Chicago, 2000.

[5] Konstantas D, Herzog R. Continuous monitoring of vital constants for mobile users: the MobiHealth approach. EMBC 2003: 3728-3731.

[6] Tompkins WJ. Biomedical Digital Signal Processing. PTR. Prentice-Hall. 1993.

Address for correspondence

René González Fernández

Calle 202 \#1704 entre 17 y 19, Playa. La Habana, Cuba. rigonzalez@icid.cu 